\title{
INVESTIGATION OF THE OPTICAL VARIABILITY OF RELATIVISTIC OBJECTS WITH HIGH TIME RESOLUTION
}

\author{
S. N. MITRONOVA ${ }^{1}$, G. M. BESKIN ${ }^{1}$, S. I. NEIZVESTNY ${ }^{1}$ \\ V. L. PLOKHOTNICHENKO ${ }^{1}$, M. YU. POPOVA ${ }^{1}$, \\ S. ZHARIKOV ${ }^{1}$, O. G. BENVENUTO ${ }^{2}$, C. FEINSTEIN ${ }^{2}$, \\ M. MENDEZ ${ }^{2}$, V. BORIAKOFF ${ }^{3}$, D. DOSSA ${ }^{3}$ \\ 1. Special Astrophysical Observatory (SAO), Nizhnij Arkhyz, \\ Karachaevo-Cherkesia, 357147, Russia \\ 2. Observatorio Astronomico de la Plata, Paseo del Bosque \\ s/n, 1900 La Plata, Argentina \\ 3. Worcester Polytechnic Institute, USA
}

1. Detection of nonthermal optical flares in low mass X-ray binaries. Eleven low-mass X-ray binaries have been observed with the MANIA (Multichannel Analysis of Nanoseconds Intensity Alteration) complex; $\mathrm{X} 0420+32, \mathrm{~A} 0620-00, \mathrm{X} 1728-169, \mathrm{X} 1813-14, \mathrm{X} 1957+11$ in 1986 at the $6 \mathrm{~m}$ telescope of SAO [7], and 2S0921-630, 4U1543-475, 4U1636-536, 4U1559-487, MXB1735-44, 4U1822-371 in 1991 at the $2.15 \mathrm{~m}$ telescope of CASLEO (Argentina) [1]. Analysis of the data using the special $y_{2}$ and $d_{2}$ function formalism [4], [6] has shown no brightness variations on time-scales of $10^{-7} \ldots 10 \mathrm{~s}$ for all objects except A0620-00 and MXB1735-44.

A0620-00. The five millisecond flashes were detected on 1986 February 13 (Fig. 1). The first two flares have durations of $3 \mathrm{~ms}$ and $5 \mathrm{~ms}$ and their rise times were $1 \ldots 2 \mathrm{~ms}$. The other three events lasted $0.4 \ldots 0.5 \mathrm{~ms}$ with rise times of about $0.1 \mathrm{~ms}$. The lower limit of the flare amplitudes is 40 counts $\mathrm{ms}^{-1}$ and the corresponding brightness temperatures are $510^{9} \mathrm{~K}$

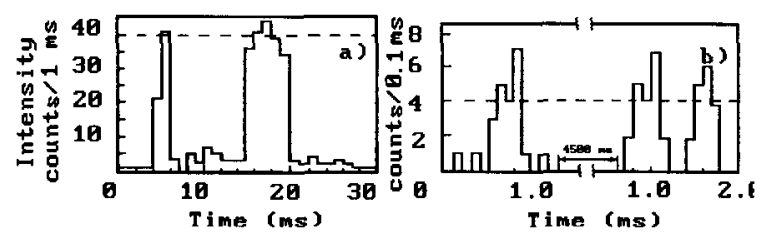

Figure 1. The ultrashort flares of A0620-00 on 1986 February 13, taken in white light with aperture 4.3 arcsec and seeing $1.5 \mathrm{arcsec}$; the dashed line marks the level of maximum intensity corresponding to the limiting transfer rate into the computer.

403

A. Evans and J. H. Wood (eds.), Cataclysmic Variables and Related Objects, 403-404.

(C) 1996 Kluwer Academic Publishers. Printed in the Netherlands. 


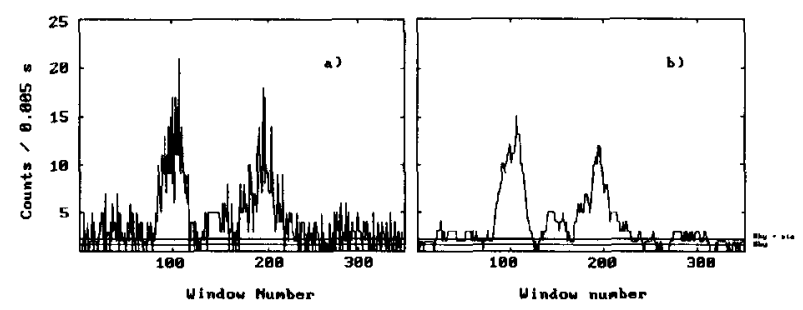

Figure 2. The white light curves (taken with aperture - 12 arcsec) of two flares of MXB1735-44. (a) Initial light curve, (b) smoothed light curve.

for the first two and $510^{11} \mathrm{~K}$ for the others.

MXB1735-44. Two flares with duration of about $0.25 \mathrm{~s}$ were detected on 1991 May 9 (Fig. 2). The rise times of these flares are $110 \mathrm{~ms}$ and $120 \mathrm{~ms}$ (about $60 \mathrm{~ms}$ at the most steep parts) and their relative amplitudes are 32 and 17. The corresponding brightness temperatures are from $410^{7}$ to $210^{8} \mathrm{~K}$. In order to study the fine structure of these events the detailed light curve, $I(t)$, was analyzed using the 'splash method' [1]. This method showed that both flares have fine structure on time-scales of $5 \ldots 10 \mathrm{~ms}$ (Fig. 2a). The lower limit on the brightness temperatures of the fine structure is $210^{10} \mathrm{~K}$. We found a distance $D \approx 1 \mathrm{kpc}, B \approx 19.3 \mathrm{mag}, A_{\mathrm{v}} \approx$ $1.2 \mathrm{mag}$ for $\mathrm{A} 0620-00$ [7] and $D \approx 7 \mathrm{kpc}, B \approx 17.2 \mathrm{mag}, A_{\mathrm{v}} \approx 0.8 \mathrm{mag}$ for MXB1735-44 [1]. Thus, the brightness temperatures of the A0620-00 and MXB1735-44 flares $\left(10^{8} \ldots 10^{11} \mathrm{~K}\right)$ contradict a thermal mechanism for their generation and support a nonthermal model.

2. A search for optical pulses of the $6.1 \mathrm{~ms}$ binary radio pulsar PSR 1953+290. The observations of the $6.1-\mathrm{ms}$ radio pulsar PSR $1953+$ 290 [2] were carried out in 1992 at the $6 \mathrm{~m}$ telescope with a CCD photometer to identify the optical counterpart of the source using its radio position and the MANIA complex (white light with 7 arcsec diaphragm) to search for optical pulses. No optical pulses or any stochastic variability [5] have been detected. The upper limit for brightness in the $B$-band is 26.5 mag with a significance level $<3 \%$. By comparing the upper limit of the pulsar optical luminosity (taking into account the distance [2]) with the Crab pulsar luminosity gives us an upper limit for the magnetic field of $B \approx 10^{8} \mathrm{G}$.

\section{References}

1. Beskin, G.M., Neizvestny, S.I., Plokhotnichenko, V.L., et al., 1994, A\&A, 289, 141

2. Boriakoff, V., Buccheri, R., Fauci, F., 1983, Nature, 304, 417

3. Elvis, M., Page, C.G., Pounds, K.A., et al., 1975, Nature, 257, 656

4. Plokhotnichenko, V.L., 1983, Soobshchenija SAO, 38, 29

5. Plokhotnichenko, V.L., 1992, Ph.D. thesis, Special Astrophysical Observatory

6. Shvartsman, V.F., 1977, Soobshchenija SAO, 19,5

7. Shvartsman, V.F., Beskin, G.M., Mitronova, S.N., et al., 1989, Sov. Ast. Let., 15, 590 